\title{
Influencia de la publicidad televisiva sobre el consumo de suplementos de testosterona
}

\author{
Influence of television advertising on consumption of testosterone supplements
}

\begin{abstract}
Objetivos
Analizar la asociación entre la publicidad directa al consumidor (DTCA) de testosterona transmitida por televisión y la iniciación de análisis y/o tratamientos de suplementación hormonal.

\section{Diseño}

Estudio ecológico que exploró entre 2009 y 2013 la asociación geográfica y temporal entre las mediciones mensuales de rating televisivo vinculado a anuncios publicitarios de concientización sobre posibilidad de tener niveles bajos de testosterona y/o de productos específicos para paliar dichos déficits; y la realización en varones mayores de 18 años de nuevos estudios hormonales y/o de iniciaciones de tratamientos con testosterona o productos vinculados, de acuerdo a los registros de sus Seguros de Salud.
\end{abstract}

\section{Lugar}

75 áreas de mercado determinadas (AMD) de Estados Unidos definidas por una exposición a una influencia televisiva similar.

\section{Medición de Resultados Principales}

Fueron medidas tres tasas de incidencia: 1) la de realización de
Layton B y col. JAMA 2017; 317(11):1159-1166. nuevos estudios de testosterona en sangre; 2 ) la de iniciación de tratamientos con cualquier producto y/o marca (inyecciones en el consultorio, implantes, etc.), 3) la de iniciación de tratamientos sin la realización previa de un análisis.

\section{Resultados Principales}

De 17.228.599 hombres asegurados en los 75 AMD, 1.007.990 (edad promedio 49,6 años) realizaron nuevos exámenes de testosterona en sangre y 283.317 (edad promedio 51,8 años) iniciaron suplementaciones de testosterona. La intensidad de la exposición varió temporal y geográficamente, registrándose la mayor en el sudeste del país. Antes de 2012 predominaron los anuncios de sensibilización respecto del descenso de la producción de testosterona vinculado con el envejecimiento; y, luego de esa fecha, los anuncios de productos de marcas específicas para la suplementación de dichos "descensos". Asumiendo un comportamiento lineal de la asociación entre la intensidad de la exposición y los tres resultados evaluados, la tabla 1 resume las incidencias absolutas de los diferentes sucesos y el aumento promedio en la probabilidades de observar alguna conducta potencialmente condicionada por la publicidad televisiva, por cada unidad de aumento en la exposición.

Tabla 1. Asociación entre la intensidad de la exposición publicitaria y la iniciación de análisis y/o tratamientos de reemplazo hormonal, con o sin estudios diagnóstico previos.

\begin{tabular}{l|c|c} 
& Tasas absolutas por cada 10 mil hombres (IC 95\%) & $\begin{array}{c}\text { Aumento relativo por cada incremento de una unidad de } \\
\text { exposición (IC 95\%) }\end{array}$ \\
\hline De alguna determinación de testosterona & $0,14(0,01 \mathrm{a} 0,19)$ & $1,006(1,004 \mathrm{a} 1,008)$ \\
\hline De iniciación de tratamiento & $0,05(0,03 \mathrm{a} 0,08)$ & $1,007(1,004 \mathrm{a} 1,010)$ \\
\hline De iniciación sin pruebas previas & $0,02(0,01 \mathrm{a} 0,03)$ & $1,008(1,002 \mathrm{a} 1,013)$ \\
\hline
\end{tabular}

Cada unidad de exposición fue calculada a través del producto entre la fracción alcanzada de la audiencia blanco (estimada a través de encuestas y mediciones electrónicas) y la carga de la exposición en términos de cantidad de avisos emitidos por unidad de tiempo. Cada aumento de una unidad de exposición corresponde aproximadamente una visualización adicional de un aviso publicitario por hogar.

\section{Conclusiones}

La exposición regional a publicidades directas transmitidas por la televisión se asoció a una mayor realización de pruebas de testosterona y de iniciación de tratamientos, con o sin análisis de referencia previos.
Fuente de financiamiento/conflicto de interés de los autores: Instituto Nacional del Envejecimiento, Instituto Nacional del Corazón, Pulmones y Sangre y Universidad de Carolina del Norte/El Dr. Alexander es presidente de un comité asesor de la FDA y se desempeña como consultor.

\section{Comentario}

El discurso publicitario es un canal de información para los ciudadanos que, de algún modo, contribuye a la construcción de imaginarios sociales, como el de la salud o del mismo cuerpo ${ }^{1}$. Por eso, es imprescindible analizar y reflexionar sobre su influencia, como es el caso de este estudio, que busca detectar el impacto del aumento de publicidades que apelan al ideal masculino del adulto fuerte, saludable y viril ${ }^{2}$. Los autores tomaron nota de que durante la última década se ha incrementado el uso de testosterona para contrarrestar los síntomas de la denominada andropausia, que suelen afectar rasgos asociados a la virilidad, produciendo pérdida de fuerza y masa muscular ${ }^{3}$, falta de deseo sexual y problemas para sostener la erección. Sin embargo, la eficacia y la seguridad de estas suplementaciones, que solo están recomendadas para el tratamiento de algunas situaciones clínicas especificas como el hipogonadismo documentado, no han sido probadas ${ }^{4}$. Más aún, el uso de estas hormonas ha sido vinculado con un aumento de riesgo de eventos cardíacos y/o cere-brovasculares, y con el aumento de la velocidad de crecimiento de tumores de próstata ${ }^{5}$. Como resultado, no solo encon- traron un vínculo entre ambas situaciones sino que también detectaron que tal promoción habría potenciado usos inapropiados de estas hormonas. Así, estos datos son un aporte para repensar los contenidos de publicidades y promociones de medicamentos en general. De hecho, existen normas y criterios éticos para promocionar productos vinculados a la salud 6 , siendo EE.UU. uno de los únicos países donde se permite la publicidad directa a los consumidores de medicamentos que requieren prescripción médica ${ }^{7}$, lo que actualmente esta tratando de ser frenado ${ }^{8,9}$.

\section{Conclusiones de la comentadora}

Este estudio representa una nueva prueba del poder de la publicidad para influenciar a las personas a que consuman medicamentos, incluso en forma inadecuada y para situaciones clínicas para las que no existe evidencia que los riesgos de consumirlos superen los beneficios. Estos resultados Ilaman a la reflexión sobre su promoción, ya que los medicamentos no son meras mercancías sino que responden a necesidades de salud pública, entendida esta como un bien social. 
Vanina Lombardi [ AgenciaTSS. Universidad de San Martín vanina.lombardi@gmail.com ]

Lombardi V. Influencia de la publicidad televisiva sobre el consumo de suplementos de testosterona. Evid Act Pract Ambul 2017;20(2): 46-47. Comentado de: Layton B y col. Association Between Direct-to-Consumer Advertising and Testosterone Testing and Initiation in the United States, 2009-2013. JAMA 2017; 317/11:1159-66. PMID: 28324090.

Referencias

1. Petracci M y col. Comunicación y salud en Argentina, Buenos Aires, La Crujía Ediciones. 2011.

2. Se pueden ver ejemplos de publicidades de testosterona televisadas en Los Estados Unidos en Health News Review: https://www.healthnewsreview.org/2017/03/testosteroneadvertising-maybe-not-the-safest-way-to-get-your-mojo-back/. Accedido en julio de 2017.

3. Biblioteca Nacional de Los Estados Unidos. MedlinePlus. Enciclopedia médica. https://medlineplus.gov/spanish/ency/patientinstructions/000722.htm. Accedido en julio de 2017 4. Administración de Alimentos y Drogas de los Estados Unidos (FDA). Seguridad y disponibilidad de drogas. FDA Drug Safety Communication: FDA cautions about using testosterone products for low testosterone due to aging; requires labeling change to inform of possible increased risk of heart attack and stroke with use. https://www.fda.gov/Drugs/ DrugSafety/ucm436259.htm Accedido en julio de 2017.

5. Ídem 3.

6. Organización Panamericana de la Salud/Organización Mundial de la Salud. Promoción de medicamentos. Disponible en http://www.paho.org/hq/index.php?option=com_cont ent\&view=article\&id=8236\%3A2013-medicines-promotion\&catid=4997\%3Apromotion\&ltemid=39823\&lang=es. Accedido en julio de 2017 .

7. Almasi $E$ y col. What are the public health effects of direct-to-consumer drug advertising? PLoS Med. 2006;3(3):e145. https://www.ncbi.nlm.nih.gov/pubmed/16563041

8. Organización mundial de la salud. Boletín. La publicidad directa al consumidor en la línea de fuego. http://www.who.int/bulletin/volumes/87/8/09-040809/es/ Accedido en julio de 2017.

9. American Medical Association. AMA calls for ban on DTC ads of prescription drugs and medical devices [press release]. November 17, 2015. https://www.ama-assn.org/content/ama-calls-ban-direct-consumer-advertising-prescription-drugs-and-medical-devices. Accedido en julio de 2017.

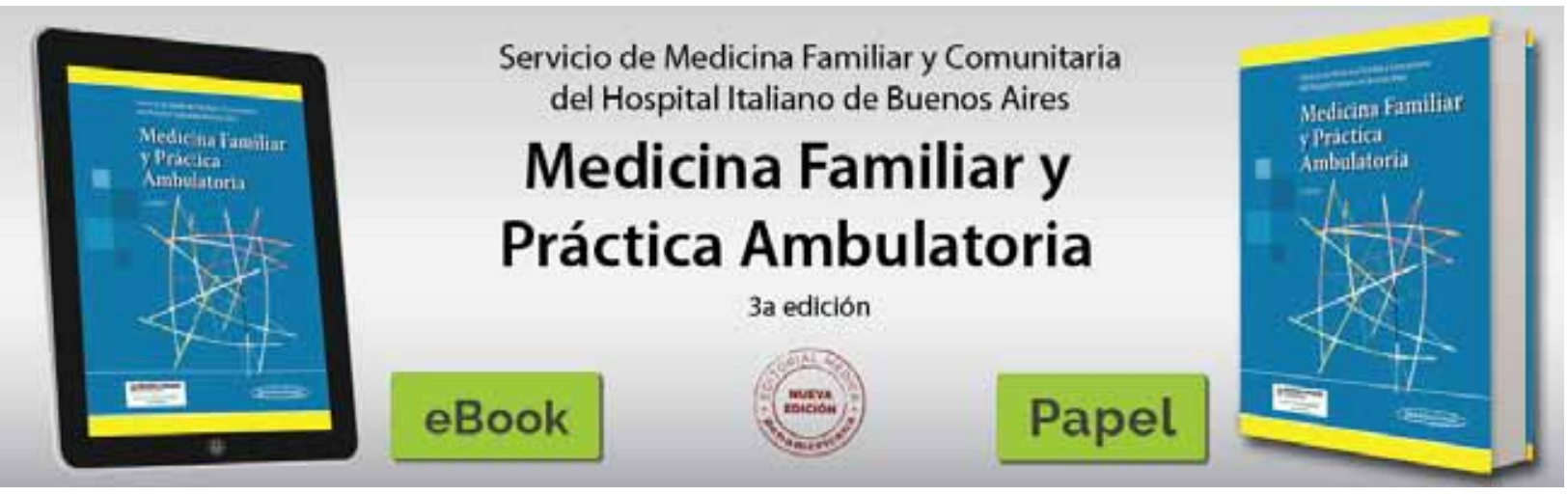

